\title{
Sugar-sweetened beverage intake and cardiovascular risk factor profile in youth with type 1 diabetes: application of measurement error methodology in the SEARCH Nutrition Ancillary Study
}

\author{
Angela D. Liese ${ }^{1 *}$, Jamie L. Crandell ${ }^{2}$, Janet A. Tooze ${ }^{3}$, Victor Kipnis ${ }^{4}$, Ronny Bell ${ }^{5}$, Sarah C. Couch ${ }^{6}$, \\ Dana Dabelea ${ }^{7}$, Tessa L. Crume ${ }^{7}$ and Elizabeth J. Mayer-Davis ${ }^{8}$ \\ ${ }^{1}$ Department of Epidemiology and Biostatistics, Center for Research in Nutrition and Health Disparities, Arnold School of \\ Public Health, University of South Carolina, 915 Greene Street, Columbia, SC 29208, USA \\ ${ }^{2}$ School of Nursing and Department of Biostatistics, University of North Carolina, Chapel Hill, NC, USA \\ ${ }^{3}$ Department of Biostatistical Sciences, Wake Forest School of Medicine, Winston-Salem, NC, USA \\ ${ }^{4}$ Biometry, Division of Cancer Prevention, National Cancer Institute, Rockville, MD, USA \\ ${ }^{5}$ Department of Epidemiology and Prevention, Wake Forest School of Medicine, Winston-Salem, NC, USA \\ ${ }^{6}$ Department of Nutritional Sciences, University of Cincinnati Medical Center, Cincinnati, OH, USA \\ ${ }^{7}$ Department of Epidemiology, Colorado School of Public Health, University of Colorado, Denver, Aurora, CO, USA \\ ${ }^{8}$ Departments of Nutrition and Medicine, University of North Carolina, Chapel Hill, NC, USA
}

(Submitted 21 October 2014 - Final revision received 10 April 2015 - Accepted 27 May 2015 - First published online 16 July 2015)

\begin{abstract}
The SEARCH Nutrition Ancillary Study aims to investigate the role of dietary intake on the development of long-term complications of type 1 diabetes in youth, and capitalise on measurement error (ME) adjustment methodology. Using the National Cancer Institute (NCI) method for episodically consumed foods, we evaluated the relationship between sugar-sweetened beverage (SSB) intake and cardiovascular risk factor profile, with the application of ME adjustment methodology. The calibration sample included 166 youth with two FFQ and three $24 \mathrm{~h}$ dietary recall data within 1 month. The full sample included 2286 youth with type 1 diabetes. SSB intake was significantly associated with higher TAG, total and LDL-cholesterol concentrations, after adjusting for energy, age, diabetes duration, race/ethnicity, sex and education. The estimated effect size was larger (model coefficients increased approximately 3-fold) after the application of the NCI method than without adjustment for ME. Compared with individuals consuming one serving of SSB every 2 weeks, those who consumed one serving of SSB every $2 \mathrm{~d}$ had $3.7 \mathrm{mg} / \mathrm{dl}(0.04 \mathrm{mmol} / \mathrm{l})$ higher TAG concentrations and $4.0 \mathrm{mg} / \mathrm{dl}(0.10 \mathrm{mmol} / \mathrm{l})$ higher total cholesterol and LDL-cholesterol concentrations, after adjusting for ME and covariates. SSB intake was not associated with measures of adiposity and blood pressure. Our findings suggest that SSB intake is significantly related to increased lipid levels in youth with type 1 diabetes, and that estimates of the effect size of SSB on lipid levels are severely attenuated in the presence of ME. Future studies in youth with diabetes should consider a design that will allow for the adjustment for ME when studying the influence of diet on health status.
\end{abstract}

Key words: FFQ validation: Reliability: Youth: Diabetes mellitus

Not much is known about the role of nutrition in the development of CVD risk in youth with diabetes ${ }^{(1,2)}$. Understanding the role of nutrition in the development of diabetes complications is, however, fraught with challenges. The complexities of assessing the diet can lead to biased estimates of the relationship between self-reported usual intake and health outcomes. In parallel with ongoing efforts to improve dietary assessment, statistical analysis methods have advanced to adjust for the effects of dietary measurement error (ME) in studies of usual dietary intake and health outcomes ${ }^{(3,4)}$. The method of choice in nutritional epidemiology is regression calibration, which requires a subsample with an unbiased measure of dietary intake in addition to a dietary assessment instrument used in the main study, such as a FFQ. The subsample is used to estimate predicted values of true intake (i.e. its conditional expectation), given FFQ measurements and other covariates into account in the risk model. Using those predicted values instead of unknown true intake leads

Abbreviations: ME, measurement error; NDSR, Nutrition Data System for Research; SNAS, SEARCH Nutrition Ancillary Study; SSB, sugar-Sweetened beverages; WHtR, waist:height ratio.

*Corresponding author: A. D. Liese, fax +1 803777 2524, email liese@sc.edu 
to approximately consistent estimates of regression parameters in the risk model, reflecting the relationship between dietary intake and health outcomes.

Intake of sugar-sweetened beverages (SSB) is a high-profile topic in public health policy and research and particularly relevant for individuals with diabetes ${ }^{(5,6)}$. We have previously shown that high SSB intake as assessed by FFQ is associated with higher levels of total cholesterol, LDL-cholesterol and plasma TAG in youth with type 1 diabetes in the SEARCH for Diabetes in Youth Study ${ }^{(7)}$. However, FFQ are known to be prone to substantial ME, which results in the usually attenuated estimate of dietary effect and loss of statistical power to detect the effect ${ }^{(8)}$. The recent completion of a diet assessment and calibration sub-study in the same study allowed us to re-evaluate the previous findings. Thus, the purpose of the present study was to obtain estimates of the association between SSB intake and lipids levels in the SEARCH Study, after adjusting for FFQ ME using the regression calibration method. We further extended the analysis to include measures of adiposity and blood pressure as additional outcomes.

\section{Research design and methods}

\section{SEARCH study design}

SEARCH is a multi-centre study that began conducting population-based ascertainment of non-gestational cases of physician-diagnosed diabetes in youth $<20$ years of age in 2001. The study ascertained prevalent cases in 2001 and 2009 and incident cases starting from 2002 through to the present $^{(9)}$. The protocol was compliant with the Health Insurance Portability and Accountability Act and approved by the local institutional review boards. Case ascertainment was conducted using a network of health care providers including paediatric endocrinologists, hospitals and other providers. Individuals with diabetes if they were $<18$ years of age or their parent/guardian were invited to complete a brief survey. Those whose diabetes was not secondary to other health conditions were invited to the baseline study visit, which included questionnaires, physical examinations and laboratory measurements. Diabetes type, as assigned by the health care provider, was categorised as type 1, type 2 and other type (including hybrid type, maturity onset of diabetes in youth, type designated as 'other', type unknown by the reporting source, and missing).

\section{Data collection in SEARCH}

Dietary intake was assessed with a FFQ (available upon request), which was modified from the Block Kids Questionnaire with an expanded list of foods selected to consider ethnic, cultural and regional diversity ${ }^{(10)}$. The FFQ was completed by participants aged 10 years and older and generally by the youth without assistance after receiving instructions from staff. It consisted of eighty-five food lines for which the participant indicated whether the item(s) was/were consumed in the past week ('yes/no') and if yes, the participants were asked to indicate the number of days and the average portion size for each food consumed. SSB intake was aggregated from four questions that asked about the intake of (1) soda (Coke, Sprite $^{\circledR}$, etc., not counting diet soda), (2) Kool-Aid and Gatorade, (3) Sunny Delight ${ }^{\circledR}$, Hi-C-C ${ }^{\circledR}$, Hawaiian Punch ${ }^{\circledR}$ and Ocean Spray ${ }^{\circledR}$, and (4) coffee or tea sweetened with sugar. Participants were asked to report the number of glasses, juice boxes or cups usually consumed in a day for each beverage category. An open-ended question at the end of the FFQ asked about other foods that a participant might wish to report. The nutrient and portion size databases for this instrument were modified from the respective Diabetes Prevention Program databases, using the Nutrition Data System for Research (NDSR, Nutrition Coordinating Center, University of Minnesota, Minneapolis, MN, database version 2.6/8A/23) and industry sources. Daily energy intake was estimated by aggregating across all foods reported on the FFQ.

Lipid levels were determined in plasma samples taken during the in-person visits, and specimens were processed at the site and shipped within $24 \mathrm{~h}$ to the Northwest Lipid Metabolism and Diabetes Research Laboratories in Seattle, Washington. Measurements of plasma cholesterol, TAG, and HDL-cholesterol concentrations were performed on a Hitachi 917 autoanalyser (Boehringer Mannheim Diagnostics). LDLcholesterol concentration was calculated by the Friedewald equation for individuals with TAG concentration $<400 \mathrm{mg} / \mathrm{dl}$ $(<4.52 \mathrm{mmol} / \mathrm{l})$ and by the beta-quantification procedure for those with TAG concentration $\geq 400 \mathrm{mg} / \mathrm{dl}(\geq 4.52 \mathrm{mmol} / \mathrm{l})^{(11)}$.

Height was measured twice (in $\mathrm{cm}$ ) using a stadiometer. Waist circumference was assessed according to the National Health and Nutrition Examination Survey protocol as the circumference just above the right iliac crest at the mid-axillary line ${ }^{(12)}$. A measuring tape was used and two measurements were taken. If the first two measures differed by $>1.0 \mathrm{~cm}$ for waist circumference or $>0.5 \mathrm{~cm}$ for height, a third measurement was taken. The average of the two or three measurements was used for analyses. The waist:height ratio (WHtR) was calculated by dividing the average waist circumference by the average height. Weight was measured (in $\mathrm{kg}$ to the nearest $0 \cdot 1 \mathrm{~kg}$ ) using an electronic scale. BMI was calculated for each participant and converted to an age- and sex-specific BMI $z$-score according to the Centers for Disease Control and Prevention guidelines.

Systolic and diastolic blood pressure measurements were taken three times using a portable mercury sphygmomanometer, with a cuff chosen to fit the size of the participant's arm. The average of the three readings was used for analyses.

Other data collected from the parent included the following information used as covariates in the present analyses: date of birth, sex, parental education and date of diabetes diagnosis were obtained through self-report; age and diabetes duration were calculated; race and ethnicity were obtained using the standard census questions ${ }^{(13)}$

The present analysis was restricted to youth with type 1 diabetes whose diabetes was prevalent in 2001 or incident in 2002-2005 and who were $\geq 10$ years of age at the time of their baseline study visit. Of the 2792 participants who met these criteria, 2304 had diabetes for at least 3 months at the time of their study visit and additionally had complete 
data on fasting lipid levels, FFQ and covariates. An additional eighteen participants were excluded due to evidence of hypertriacylglycerolaemia (fasting TAG concentration $>400 \mathrm{mg} / \mathrm{dl}(>4.52 \mathrm{mmol} / \mathrm{l})$ ), yielding a final analytic sample size of 2286. Most participants also had data available for non-lipid outcomes: WHtR ( $n$ 2208); BMI $z$-score ( $n$ 2267); systolic blood pressure and diastolic blood pressure ( $n$ 2257).

\section{Calibration sample}

The SEARCH Nutrition Ancillary Study (SNAS) was designed to examine the associations of nutritional factors with the progression of insulin secretion defects and the presence of CVD risk factors in youth with type 1 diabetes. The SNAS protocol was reviewed and approved by the institutional review boards of all participating institutions. The SNAS included a dedicated diet assessment sub-study that served as the calibration sample in the analyses of dietary intake-disease outcome relationships, adjusting for ME. The calibration sample consisted of 166 participants with FFQ data and one to three $24 \mathrm{~h}$ dietary recalls ( $152 \mathrm{had}$ three recalls, eight had two recalls, and six had one recall, for a total of 494 recalls).

The previous day $24 \mathrm{~h}$ dietary recalls were conducted by trained and certified staff of the University of North Carolina at Chapel Hill (Nutrition Obesity Research Center - Diet, Physical Activity and Body Composition Core) by telephone on randomly selected, non-consecutive days including two weekdays and one weekend day during a 4-week sampling window. NDSR version 2008 and 2009 software licensed from the Nutrition Coordinating Center at the University of Minnesota was employed, using the multi-pass approach in which a participant was first asked to provide a general listing of foods consumed on the previous day, starting with the first food consumed after awakening and ending with the last food consumed before sleep, and grouped by eating episodes. Subsequently, the interviewing dietitian reviewed the list with the participant and prompted for foods or eating episodes forgotten or omitted, queried for more detail on the time, name and location of eating episodes, collected details about the foods reported including quantity, portion size and food description, and verified the information and prompted for any omissions.

The 166 individual foods that were ascertained from the $24 \mathrm{~h}$ dietary recalls with the NDSR system were grouped into twenty-seven specific food groups, including SSB, which consisted of sweetened soda, sweetened fruit drinks, sweetened water, sweetened coffee, and sweetened tea. If the portion size units differed between the $24 \mathrm{~h}$ dietary recall and the FFQ, appropriate conversions were made to the FFQ data. We have previously shown a reasonable relative validity of the SSB food group with a correlation of $\rho_{\mathrm{QT}}=0.54$ between true and FFQ-reported intakes in a model adjusted for $\mathrm{ME}^{(14)}$.

\section{Statistical analysis}

Characteristics of the sample of SEARCH participants included in these analyses and the calibration subsample were computed using means and standard deviations or medians for continuous variables and frequencies for categorical variables.
To estimate the relationship between SSB intake and lipid levels, we performed the regression of log-transformed lipids on SSB intake, energy and covariates (diabetes duration, age, sex, race/ethnicity and parental education) in the risk model. Usual statistical analysis methods for assessing exposure-outcome relationships assume that exposure is measured without error. We used the National Cancer Institute method to apply regression calibration to FFQ-reported SSB and energy intakes to adjust for ME in the risk models relating SSB intake to lipid outcomes ${ }^{(3,8,15)}$.

An overview of this process is given here, followed by details of the approach. For both energy and SSB intakes, the $24 \mathrm{~h}$ dietary recall was assumed to be unbiased for true intake. The calibration subsample was used to build ME models for the $24 \mathrm{~h}$ dietary recall-reported intake of SSB and total energy, which included FFQ-reported intake of SSB, energy and other adjusting covariates in the risk model. The resulting model was then used to predict the true usual intake of SSB and energy from the intakes of SSB and energy measured by the FFQ and covariates. Subsequently, the predicted true usual SSB intake and covariates were used in a regression model for blood lipids in the SEARCH sample.

We used a two-part model (the National Cancer Institute method) to account for the fact that SSB was consumed episodically, i.e. not consumed by all participants every day ${ }^{(16)}$. This requires a special methodology in specifying the ME model for SSB intake reported on the $24 \mathrm{~h}$ dietary recalls. Part I of the model predicts the probability of SSB intake on any given day using mixed-effects logistic regression, and part II of the model predicts the daily amount of SSB consumed using a mixed-effects linear model on a transformed scale. The transformation is chosen so that the person-specific random effect and the within-person random error are approximately normally distributed. Both parts of the model are fitted simultaneously, allowing person-specific random effects to be correlated $^{(16)}$. Energy intake is not episodic, so a simpler model (without the probability of intake component) was appropriate ${ }^{(4)}$.

In the calibration sample, the $24 \mathrm{~h}$ dietary recall data were modelled as a function of the FFQ data for SSB (episodic) and energy (non-episodic) separately. FFQ-reported energy intake $(\mathrm{kcal} / \mathrm{d})$ was log-transformed to improve normality, and FFQ-reported SSB intake (servings/d) was log-transformed after imputing zero intake as 0.01 servings/d. The log transformation of SSB intake effectively transformed non-zero amounts to approximate normality, but $61 \%$ of the calibration sample reported no FFQ-reported intake of SSB, making it very difficult to transform the FFQ-based estimate of intake to approximate normality. Consequently, the ME model was expanded by adding a binary $(0 / 1)$ indicator of SSB intake on the FFQ to the model. The use of two variables to capture FFQ-reported SSB intake adds flexibility to the model, estimating a separate intercept for non-consumers and a separate regression slope relating consumption amount to usual intake for consumers. In addition to the three FFQ variables, each model included diabetes duration, age, race/ethnicity, sex and parental education. Then, the 
calibration predictor models of true SSB and energy intakes were estimated from the corresponding ME models. For each participant in the full sample ( $n$ 2286), the developed models were used to obtain the regression calibration predictor of each participant's usual SSB and energy intakes, conditional on the observed FFQ and the other model covariates $^{(3)}$.

The predicted SSB (servings/d) and energy values from the calibration models were used in a linear regression risk model, controlling for the same covariates that were in the calibration model. Blood lipid outcomes were log-transformed to improve the model fit, and other outcomes were left untransformed. To account for the fact that regression calibration predictors were estimated in a subsample, the standard errors in the disease model were calculated using the bootstrap method. All statistical analyses were conducted using SAS 9.2 (SAS Institute, Inc.).

\section{Results}

The primary study sample comprised 2286 youth with type 1 diabetes, with a mean age just below 15 years at the time of their baseline study visit (range 10-22; Table 1 ). The majority (77\%) were non-Hispanic white, 50\% were female and $47 \%$ had at least one parent with a college degree. Table 1 also shows comparisons between the study sample and calibration sample. The two groups were similar with respect to sex and race/ethnicity. However, the calibration sample, which consisted of prevalent type 1 cases, had a much longer duration of diabetes than the overall sample, which included incident as well as prevalent cases. The calibration sample was also slightly older at the study visit, and their parents were slightly more educated.

Descriptive statistics for cardiovascular risk factor outcomes and FFQ predictors for the SEARCH sample and the calibration

Table 1. Descriptive characteristics of the study sample and the calibration sample in the SEARCH Nutrition Ancillary Study

(Number of participants and percentages; mean values and standard deviations or ranges)

\begin{tabular}{|c|c|c|c|c|}
\hline \multirow[b]{2}{*}{ Variables } & \multicolumn{2}{|c|}{$\begin{array}{l}\text { Total sample } \\
\text { (n 2286) }\end{array}$} & \multicolumn{2}{|c|}{$\begin{array}{l}\text { Calibration } \\
\text { sample } \\
(n 166)\end{array}$} \\
\hline & $n$ & $\%$ & $n$ & $\%$ \\
\hline \multicolumn{5}{|l|}{ Age (years) } \\
\hline Mean & \multicolumn{2}{|c|}{$14 \cdot 8$} & \multicolumn{2}{|c|}{$16 \cdot 2$} \\
\hline SD & \multicolumn{2}{|c|}{$3 \cdot 0$} & \multicolumn{2}{|c|}{3.5} \\
\hline \multicolumn{5}{|l|}{ Duration of diabetes (months) } \\
\hline Mean & \multicolumn{2}{|c|}{$55 \cdot 6$} & \multicolumn{2}{|c|}{$71 \cdot 2$} \\
\hline SD & \multicolumn{2}{|c|}{$50 \cdot 5$} & \multicolumn{2}{|c|}{$11 \cdot 0$} \\
\hline Range & \multicolumn{2}{|c|}{$3-245$} & \multicolumn{2}{|c|}{$38-96$} \\
\hline Female sex & 1138 & $50 \cdot 2$ & 83 & $49 \cdot 7$ \\
\hline \multicolumn{5}{|l|}{ Race/ethnicity } \\
\hline Black/African American & 189 & $8 \cdot 3$ & 26 & $15 \cdot 7$ \\
\hline Non-Hispanic white/Caucasian & 1753 & $76 \cdot 7$ & 122 & $73 \cdot 5$ \\
\hline Other & 344 & $15 \cdot 0$ & 18 & $10 \cdot 8$ \\
\hline \multicolumn{5}{|l|}{ Highest level of parental education } \\
\hline High school or less & 461 & $20 \cdot 2$ & 22 & $13 \cdot 3$ \\
\hline Some college/associate's degree & 743 & $32 \cdot 5$ & 62 & $37 \cdot 4$ \\
\hline Bachelor's degree or more & 1082 & $47 \cdot 3$ & 82 & $49 \cdot 4$ \\
\hline
\end{tabular}

sample are given in Table 2 . The samples were similar with respect to all outcomes of interest. Interestingly, the distribution of the SSB FFQ contained a large number of zeroes, as non-consumption of SSB on the FFQ was reported in 45 and $39 \%$ of the SEARCH and calibration samples, respectively. Table 2 also includes the predicted intakes for the SEARCH sample. Many predicted SSB intakes were quite low, with the 25 th percentile occurring at about 0.07 servings/d (about one serving of SSB every 2 weeks), and the median occurring at 0.16 servings/d (about one serving of SSB every $6 \mathrm{~d})$. It is worth noting that the median intakes of SSB and energy predicted by the ME model in the SEARCH sample were both higher than the FFQ-estimated intakes, suggesting that the FFQ was under-reporting total intake.

Table 3 presents the associations of SSB intake with cardiovascular risk factors from a naive model without adjustment for ME and a ME-adjusted model. Adjusted for age, diabetes duration, race/ethnicity, sex, and energy intake, SSB intake was associated with higher levels of plasma TAG $(P=0.03)$, total cholesterol $(P=0.04)$ and LDL-cholesterol $(P=0.01)$ in the naive model and with total cholesterol $(P=0.03)$ and LDL-cholesterol $(P=0.007)$ in the ME-adjusted model, while the association with TAG lost statistical significance $(P=0 \cdot 07)$. SSB intake was not significantly associated with any of the other outcomes (BMI $z$-score, WHtR, systolic blood pressure and diastolic blood pressure) in either the naive or ME-adjusted model.

To further understand the nature of the significant findings, we compared two levels of SSB intake, specifically one serving of SSB every $2 \mathrm{~d}(0.5$ servings/d) and one serving of SSB every 2 weeks ( 0.07 servings $/ \mathrm{d})$. This difference corresponded roughly to the 80th and 20th percentiles of predicted conditional mean intake in the SEARCH sample, or about the 70th and 30th percentiles of FFQ-reported intake in the SEARCH sample. According to the naive model (without adjustment for ME), an individual with the higher intake had, on average, TAG levels that were $2 \cdot 1 \%$ higher (i.e. log-TAG $0 \cdot 020$ higher, calculated as $0.0103 \times(\ln (0 \cdot 5)-\ln (0 \cdot 07)))$, cholesterol levels that were $0.8 \%$ higher (i.e. log-total cholesterol 0.008 higher), and LDL-cholesterol levels that were $1.4 \%$ higher (i.e. log-LDL-cholesterol 0.014 higher). After adjustment for $\mathrm{ME}$, these estimated mean differences increased so that an individual with the higher intake had, on average, $5 \cdot 2 \%$ higher TAG concentrations, $2 \cdot 3 \%$ higher total cholesterol concentrations and $4 \cdot 2 \%$ higher LDL-cholesterol concentrations.

Because of the log transformation of the outcome, estimated values of the outcomes in original units are dependent on other model covariates. To better understand the clinical implications of SSB intake, Table 4 presents estimated lipid levels for two intake levels of SSB (0.5 and 0.07 servings/d) for an individual with average values on all other model covariates. Without adjustment for ME, the difference in SSB intake accounted for a difference in each of the lipid levels of about $1.3-1.4 \mathrm{mg} / \mathrm{dl} \quad(0.02-0.03 \mathrm{mmol} / \mathrm{l})$. After adjustment for $\mathrm{ME}$, this difference increased almost 3-fold to $3 \cdot 7-4 \cdot 0 \mathrm{mg} / \mathrm{dl}(0 \cdot 04-0 \cdot 10 \mathrm{mmol} / \mathrm{l})$. 
Table 2. Distribution of cardiovascular risk factors and sugar-sweetened beverage (SSB) intake of the SEARCH sample and the calibration sample in the SEARCH Nutrition Ancillary Study

(Median values and interquartile ranges (IQR); number of participants and percentages)

\begin{tabular}{|c|c|c|c|c|}
\hline \multirow[b]{2}{*}{ Variables } & \multicolumn{2}{|c|}{$\begin{array}{l}\text { SEARCH sample } \\
\quad(n 2286)\end{array}$} & \multicolumn{2}{|c|}{$\begin{array}{c}\text { Calibration sample } \\
\text { (n } 166 \text { participants, } 494 \\
24 \mathrm{~h} \text { dietary recalls) }\end{array}$} \\
\hline & Median & IQR & Median & IQR \\
\hline TAG (mg/dl) & 67 & $50-96$ & 68 & $53-102$ \\
\hline TAG (mmol/l) & 0.76 & $0.57-1.08$ & 0.77 & $0.60-1.15$ \\
\hline Total cholesterol (mg/dl) & 165 & $145-186$ & 166 & $147-185$ \\
\hline Total cholesterol (mmol/l) & $4 \cdot 27$ & $3.76-4.82$ & $4 \cdot 30$ & $3.81-4.79$ \\
\hline LDL-cholesterol (mg/dl) & 95 & $80-113$ & 94 & $75-114$ \\
\hline LDL-cholesterol (mmol/l) & 2.46 & $2.07-2.93$ & 2.43 & $1.94-2.95$ \\
\hline BMI $z$-score & 0.66 & $0.06-1 \cdot 25$ & 0.67 & $0 \cdot 13-1 \cdot 20$ \\
\hline Waist:height ratio & 0.47 & $0.44-0.52$ & 0.48 & $0.45-0.52$ \\
\hline Systolic blood pressure & 107 & $99-113$ & 107 & $100-115$ \\
\hline Diastolic blood pressure & 68 & $61-73$ & 70 & $63-76$ \\
\hline FFQ-reported SSB intake (servings/d) & 0.14 & $0.0-0.57$ & 0.29 & $0-1$ \\
\hline FFQ-reported energy intake (kcal/d) & 1764 & $1358-2341$ & 1473 & $1127-2115$ \\
\hline FFQ-reported energy intake (kJ/d) & 7381 & $5682-9795$ & 6163 & $4715-8849$ \\
\hline \multicolumn{5}{|l|}{ Non-consumption of SSB on the FFQ } \\
\hline$n$ & \multicolumn{2}{|c|}{1020} & \multicolumn{2}{|c|}{65} \\
\hline$\%$ & \multicolumn{2}{|c|}{44.6} & \multicolumn{2}{|c|}{$39 \cdot 2$} \\
\hline \multirow{2}{*}{\multicolumn{5}{|c|}{$\begin{array}{l}24 \mathrm{~h} \text { dietary recalls } \\
\text { No SSB consumption }\end{array}$}} \\
\hline & & & & \\
\hline$n$ & \multicolumn{2}{|c|}{-} & \multicolumn{2}{|c|}{383} \\
\hline$\%$ & \multicolumn{2}{|c|}{ - } & \multicolumn{2}{|c|}{$77 \cdot 5$} \\
\hline Predicted SSB intake based on the ME adjustment model (servings/d) & 0.16 & $0.07-0.40$ & - & - \\
\hline Predicted energy intake based on the ME adjustment model (kcal/d) & 1967 & $1764-2217$ & - & - \\
\hline Predicted energy intake based on the ME adjustment model (kJ/d) & 8230 & $7381-9276$ & - & - \\
\hline
\end{tabular}

ME, measurement error.

\section{Discussion}

The present study expands on our previous report on the association of SSB intake with lipid levels in the SEARCH population $^{(7)}$ in two distinct and important ways. First, the present analysis uses a state-of-the-art statistical approach to adjust for the influence of $\mathrm{ME}{ }^{(3,15,16)}$. As with the previous study, we found that SSB intake was significantly associated with lipid levels and not with blood pressure or measures of adiposity. Compared with individuals who consumed one serving of SSB every 2 weeks, those who consumed one serving of SSB every $2 \mathrm{~d}$ had $3.7 \mathrm{mg} / \mathrm{dl}(0.04 \mathrm{mmol} / \mathrm{l})$ higher TAG concentrations and $4.0 \mathrm{mg} / \mathrm{dl}(0.10 \mathrm{mmol} / \mathrm{l})$ higher total and LDL-cholesterol concentrations, after adjusting for ME. Second, built into the measurement error adjustment approach was a more appropriate modelling of the episodic nature of SSB intake. In contrast, the previous study had utilised a categorical approach to exposure classification without consideration of ME. The present results underscore the importance of adjusting for ME related to dietary exposure assessment.

Table 3. Associations of sugar-sweetened beverage (SSB) intake and cardiovascular risk factors in youth with type 1 diabetes aged $\geq 10$ years, with and without adjustment for measurement error (ME), in the SEARCH Nutrition Ancillary Study*

( $\beta$-Coefficients and standard deviations)

\begin{tabular}{|c|c|c|c|c|c|c|}
\hline \multirow[b]{3}{*}{ Outcomes } & \multicolumn{3}{|c|}{$\begin{array}{l}\text { Naive model with no } \\
\text { adjustment for ME }\end{array}$} & \multicolumn{3}{|c|}{ ME-adjusted model } \\
\hline & \multicolumn{3}{|c|}{ Log-SSB intake from the FFQ } & \multicolumn{3}{|c|}{ Log-predicted mean SSB intake } \\
\hline & $\beta$ & SE & $P$ & $\beta$ & SE & $P$ \\
\hline Log-TAG & 0.0103 & 0.0049 & 0.03 & 0.0258 & 0.0141 & 0.07 \\
\hline Log-total cholesterol & 0.0040 & 0.0019 & 0.04 & 0.0117 & 0.0054 & 0.03 \\
\hline Log-LDL-cholesterol & 0.0070 & 0.0027 & 0.01 & 0.0210 & 0.0077 & 0.007 \\
\hline BMI z-score & 0.0023 & 0.0090 & 0.80 & 0.0297 & 0.0286 & 0.30 \\
\hline Waist:height ratio & -0.0003 & 0.0003 & 0.61 & 0.0014 & 0.0020 & 0.47 \\
\hline Systolic blood pressure & -0.1290 & 0.1020 & 0.21 & -0.4227 & 0.2990 & $0 \cdot 16$ \\
\hline Diastolic blood pressure & -0.0019 & 0.0948 & 0.98 & 0.0418 & 0.2792 & 0.88 \\
\hline
\end{tabular}

* SSB intake and predicted SSB intake were measured in servings per d, before log transformation. All lipid models were adjusted for log-energy intake (based on the FFQ for the naive model and calibrated usual intake, i.e. its conditional mean true intake by taking into account FFQ intakes and other disease model covariates, for the model adjusted for measurement error), age, diabetes duration, race, sex and parental education. 
Without adjustment for ME, the magnitude of the difference in lipid levels between individuals consuming $0.07 v .0 .5$ servings of SSB per $\mathrm{d}$ would have been underestimated, by about $2 \cdot 3-2 \cdot 7 \mathrm{mg} / \mathrm{dl}(0 \cdot 02-0 \cdot 07 \mathrm{mmol} / \mathrm{l})$.

After adjustment for ME, the regression slopes for SSB intake were all higher compared with the naive model, as expected. These slopes reflect adjustment for bias in the FFQ, giving a more accurate estimate of the impact of SSB on the outcomes. However, regression calibration generally does not restore statistical power lost due to $\mathrm{ME}$, and the null associations of SSB intake and measures of adiposity and blood pressure (both before and after adjustment for ME) are good examples. In fact, in many cases, loss of statistical power due to ME may be somewhat exacerbated since regression calibration predictors are estimated in a finite subsample. For log-TAG, although the magnitude of the slope was increased, the standard error was large, leading to a slight increase in the standard error of the estimate, compared with the naive model, and loss of statistical significance of the association. This same loss of power was not observed for total and LDL-cholesterol, which may be due to the fact that: (1) regression calibration for SSB intake is highly non-linear, which may actually improve power loss, and/or (2) the ME model used to calibrate SSB intake also included an indicator of FFQ-reported SSB consumption, which is not included in these risk models, thereby leading to extended regression calibration that may increase power compared with the naive risk model ${ }^{(3)}$.

There are a number of limitations to the present study. Due to the sampling procedure used, participants in the calibration sample differed from those in the SEARCH sample with respect to a number of attributes. This sampling difference requires the assumption that, although the samples were not from the same underlying population, they have the same ME models for SSB and energy intakes. The largest demographic differences were in age and disease duration, both of which were included as covariates in the ME model, providing some degree of control for this difference. The two samples were similar with respect to the disease outcomes, but the calibration sample reported higher SSB intake and lower overall energy intake on the FFQ. While there is some overlap in terms of individuals between the SEARCH sample and the calibration sample ( $n$ 78), there is temporal differentiation in the sense that baseline data were used from the SEARCH sample, while calibration data were obtained at a follow-up visit. Lastly, similar to other studies, we relied on $24 \mathrm{~h}$ dietary recalls as the reference instrument in the ME adjustment method. This was done under the assumption that $24 \mathrm{~h}$ dietary recalls provide an unbiased estimate of true intake, which is a necessary assumption for these models, even though we recognise that the $24 \mathrm{~h}$ dietary recall is somewhat biased for protein, energy and protein density in adults $^{(17,18)}$.

The SEARCH FFQ queried dietary intake in the preceding week, because most children and youth would not have been able to cognitively integrate dietary intake over a whole year. Thus, the estimate of usual intake of SSB may have been underestimated. According to national data, about 
$36 \%$ of youth do not consume SSB, 33\% consume one serving of SSB per $\mathrm{d}$ and $31 \%$ two or more servings of SSB per $\mathrm{d}^{(5)}$. Our sample of youth with type 1 diabetes had a lower intake with $45 \%$ of youth not reporting any SSB intake, $41 \%$ one serving of SSB per $d$ and only $14 \%$ consuming two or more servings of SSB per $d$. While consumption of SSB is generally not recommended for any population because of the lack of nutritional value and high sugar content, youth with diabetes are particularly discouraged from consuming SSB in the context of carbohydrate-counting approaches. Occasionally, however, youth with diabetes will specifically use SSB to manage low blood sugar levels. The present study was not able to distinguish between these SSB uses; however, there is no reason to believe that SSB would have a differential impact on lipid levels depending on ambient blood sugar levels.

Although many of the SSB intakes predicted by the ME model were quite low, our model did not explicitly allow for never consumers. There is a model available for this purpose, extending the two-part National Cancer Institute method to allow for a probability of never having consumed. However, our calibration sample participants completed three or fewer $24 \mathrm{~h}$ dietary recalls, and convergence of the three-part model would be questionable without more $24 \mathrm{~h}$ dietary recalls available $^{(3)}$. We considered labelling those who reported no SSB intake on the FFQ as never consumers, rather than including them in the ME model; however, additional analyses revealed that $27 \%$ of FFQ non-consumers reported SSB intake in at least one of the three $24 \mathrm{~h}$ dietary recalls, suggesting that this assumption would be quite poor.

In youth aged $>10$ years enrolled in the SEARCH study, average lipid levels for youth with type 1 diabetes were elevated with a mean total cholesterol concentration of $174 \mathrm{mg} / \mathrm{dl}(4.51 \mathrm{mmol} / \mathrm{l})$, LDL-cholesterol concentration of $102 \mathrm{mg} / \mathrm{dl}(2.64 \mathrm{mmol} / \mathrm{l})$, HDL-cholesterol concentration of $55 \mathrm{mg} / \mathrm{dl}(1.42 \mathrm{mmol} / \mathrm{l})$ and TAG concentration of $91 \mathrm{mg} / \mathrm{dl}$ $(1.03 \mathrm{mmol} / \mathrm{l})^{(19)}$. Among the youth with type 1 diabetes in poor glycaemic control, $35 \%$ had high concentrations of total cholesterol $(\geq 200 \mathrm{mg} / \mathrm{dl}(5 \cdot 17 \mathrm{mmol} / \mathrm{l})), 27 \%$ had elevated concentrations of LDL-C $(\geq 130 \mathrm{mg} / \mathrm{dl}(3.36 \mathrm{mmol} / \mathrm{l}))$ and $12 \%$ had high concentrations of TAG $(\geq 200 \mathrm{mg} / \mathrm{dl}$ $(2.26 \mathrm{mmol} / \mathrm{l}))$, suggesting possible early influences on macro- and microvascular disease risks ${ }^{(19)}$. This profile is all the more concerning because CVD risk factors track from childhood to adulthood, and predict adult target organ damage ${ }^{(20-24)}$. Thus, an adverse risk profile in youth with diabetes may magnify the already 3-fold excess risk for CVD mortality associated with diabetes in adulthood ${ }^{(25)}$.

In this context of generally elevated lipid levels in youth with type 1 diabetes, the association of SSB intake with increased levels of total and LDL-cholesterol is particularly important. It is also consistent with the results from experimental studies showing the impact of high-sucrose $\operatorname{diets}^{(26,27)}$ on cholesterol. SSB also contain a significant amount of fructose (either bound to glucose within the sucrose molecule or in the free form), and we have previously shown positive associations of fructose ${ }^{(28)}$ and SSB intake ${ }^{(7)}$ with TAG concentrations. In the present analysis, SSB intake was significantly positively associated with TAG concentrations $(P=0.03)$ in the naive model (with no adjustment for ME); however, after adjustment for ME, while the magnitude of the association became larger, the new $P$ value (0.07) was short of statistical significance, probably for the reasons that we outlined above.

Even though the last decades have witnessed enormous improvements in the medical treatment of diabetes, the adverse lipid profile suggests that the identification of novel behavioural strategies for reducing CVD risk factors in youth with type 1 diabetes is particularly important. Our research group and others suggest that, similar to non-diabetic peers, dietary intake in youth with diabetes falls dramatically short of current recommendations ${ }^{(10,29)}$. Our findings that SSB intake was positively associated with lipid levels suggest that this particular dietary behaviour may be one of several promising behavioural approaches, alongside intensified glucose control, that should be further evaluated in clinical trials ${ }^{(30)}$. In non-diabetic populations, there is now growing evidence that SSB intake is associated with elevated lipid levels ${ }^{(31)}$.

Under the rubric of nutrition for children and adolescents with type 1 diabetes, the 2005 American Diabetes Association position statement on care concluded that 'nutrition recommendations are based on requirements for all (...) because there is no research on the nutrient requirements for children and adolescent with diabetes'(2). The present results aim to contribute to this gap in knowledge, and underscore the importance of adjusting for ME related to dietary exposure assessment in the analyses of the associations between diet and health outcomes. Future studies in youth with diabetes - be they observational studies evaluating nutritional risk factors or clinical trials of dietary interventions measuring adherence to dietary regimens - should be designed in a manner that will allow for the consideration of ME, because without this adjustment, associations between dietary intake and health outcomes may be severely underestimated.

The contents of this paper are solely the responsibility of the authors and do not necessarily represent the official views of the National Institute of Diabetes and Digestive and Kidney Diseases or the National Institutes of Health or the Centers for Disease Control and Prevention.

\section{Acknowledgements}

The SNAS was funded by 5R01DK077949 (Mayer-Davis, principal investigator). The authors also acknowledge the staff at the UNC Nutrition Obesity Research Center - Diet, Physical Activity and Body Composition Core (DK-56350) who conducted the $24 \mathrm{~h}$ dietary recall interviews. The SEARCH for Diabetes in Youth Study is indebted to the many youth and their families, and their health care providers, whose participation made this study possible.

The SEARCH for Diabetes in Youth Study was funded by the Centers for Disease Control and Prevention (PA no. 00097 , DP-05-069 and DP-10-001) and supported by the National Institute of Diabetes and Digestive and Kidney Diseases.

Site contract numbers are as follows: Kaiser Permanente Southern California (U48/CCU919219, U01 DP000246 and 
U18DP002714); University of Colorado Denver (U48/ CCU819241-3, U01 DP000247 and U18DP000247-06A1); Kuakini Medical Center (U58CCU919256 and U01 DP000245); Children's Hospital Medical Center (Cincinnati) (U48/ CCU519239, U01 DP000248 and 1U18DP002709); University of North Carolina at Chapel Hill (U48/CCU419249, U01 DP000254 and U18DP002708); University of Washington School of Medicine (U58/CCU019235-4, U01 DP000244 and U18DP002710-01); Wake Forest University School of Medicine (U48/CCU919219, U01 DP000250 and 200-2010-35 171).

The authors acknowledge the involvement of General Clinical Research Centers at the South Carolina Clinical and Translational Research Institute, at the Medical University of South Carolina (NIH/NCRR grant no. UL1RR029882); Seattle Children's Hospital (NIH CTSA Grant UL1 TR00423 of the University of Washington); University of Colorado Pediatric Clinical and Translational Research Center (grant no. UL1 TR000154); the Barbara Davis Center at the University of Colorado at Denver (DERC NIH P30 DK57516); the National Center for Research Resources and the National Center for Advancing Translational Sciences, National Institutes of Health (through Grant 8 UL1 TR000077); and the Children with Medical Handicaps Program managed by the Ohio Department of Health.

The authors' contributions are as follows: A. D. L. wrote the manuscript; J. L. C. and J. A. T. analysed the data, drafted the statistical analysis section, and reviewed and edited the manuscript; V. K. reviewed and edited the manuscript, and contributed to the discussion; R. B., S. C. C., D. D., T. L. C. and E. J. M. reviewed and edited the manuscript. All authors read and approved the final manuscript.

There are no conflicts of interest.

\section{References}

1. Evert AB, Boucher JL, Cypress M, et al. (2013) Nutrition therapy recommendations for the management of adults with diabetes. Diabetes Care 36, 3821-3842.

2. Silverstein J, Klingensmith G, Copeland K, et al. (2005) Care of children and adolescents with type 1 diabetes: a statement of the American Diabetes Association. Diabetes Care 28, $186-212$.

3. Kipnis V, Midthune D, Buckman DW, et al. (2009) Modeling data with excess zeros and measurement error: application to evaluating relationships between episodically consumed foods and health outcomes. Biometrics $\mathbf{6 5}$, 1003-1010.

4. Tooze JA, Kipnis V, Buckman DW, et al. (2010) A mixedeffects model approach for estimating the distribution of usual intake of nutrients: the NCI method. Stat Med 29, 2857-2868

5. Kit BK, Fakhouri TH, Park S, et al. (2013) Trends in sugarsweetened beverage consumption among youth and adults in the United States: 1999-2010. Am J Clin Nutr 98, $180-188$.

6. Han E \& Powell LM (2013) Consumption patterns of sugar-sweetened beverages in the United States. I Acad Nutr Diet 113, 43-53.

7. Bortsov AV, Liese AD, Bell RA, et al. (2011) Sugar-sweetened and diet beverage consumption is associated with cardiovascular risk factor profile in youth with type 1 diabetes. Acta Diabetol 48, 275-282.

8. Freedman LS, Schatzkin A, Midthune D, et al. (2011) Dealing with dietary measurement error in nutritional cohort studies. J Natl Cancer Inst 103, 1086-1092.

9. SEARCH Study Group (2004) SEARCH for Diabetes in Youth: a multicenter study of the prevalence, incidence and classification of diabetes mellitus in youth. Control Clin Trials 25, 458-471.

10. Mayer-Davis EJ, Nichols M, Liese AD, et al. (2006) Dietary intake among youth with diabetes: the SEARCH for Diabetes in Youth Study. J Am Diet Assoc 106, 689-697.

11. Hainline A Jr, Miller DT \& Mather A (1983) The Coronary Drug Project. Role and methods of the central laboratory. Control Clin Trials 4, 377-387.

12. CDC (2000) Center for Disease Control and Prevention National Health and Nutrition Examination Survey. Anthropometry Procedures Manual (Revised December 2000). pp. 3-30, 3-31. http://www.cdc.gov/nchs/data/ nhanes/bm.pdf (accessed July 2014).

13. Grieco EM \& Cassidy RC (2001) Overview of Race and Hispanic Origin: Census 2000 Brief. US Department of Commerce, Economic and Statistics Administration, US Census Bureau. http://www.census.gov/prod/2001pubs/ c2kbr01-1.pdf (accessed July 2014).

14. Liese AD, Crandell JL, Tooze JA, et al. (2015) Relative validity and reliability of a food frequency questionnaire in youth with type 1 diabetes. Public Health Nutr 18, 428-437.

15. Midthune D, Schatzkin A, Subar AF, et al. (2011) Validating an FFQ for intake of episodically consumed foods: application to the National Institutes of Health-AARP Diet and Health Study. Public Health Nutr 14, 1212-1221.

16. Tooze JA, Midthune D, Dodd KW, et al. (2006) A new statistical method for estimating the usual intake of episodically consumed foods with application to their distribution. J Am Diet Assoc 106, 1575-1587.

17. Kipnis V, Subar AF, Midthune D, et al. (2003) Structure of dietary measurement error: results of the OPEN biomarker study. Am J Epidemiol 158, 14-21.

18. Subar AF, Kipnis V, Troiano RP, et al. (2003) Using intake biomarkers to evaluate the extent of dietary misreporting in a large sample of adults: the OPEN study. Am J Epidemiol 158, $1-13$

19. Petitti DB, Imperatore G, Palla SL, et al. (2007) Serum lipids and glucose control: the SEARCH for Diabetes in Youth Study. Arch Pediatr Adolesc Med 161, 159-165.

20. Nicklas TA, von Duvillard SP \& Berenson GS (2002) Tracking of serum lipids and lipoproteins from childhood to dyslipidemia in adults: the Bogalusa Heart Study. Int $J$ Sports Med 23, Suppl. 1, S39-S43.

21. Bao W, Threefoot SA, Srinivasan SR, et al. (1995) Essential hypertension predicted by tracking of elevated blood pressure from childhood to adulthood: the Bogalusa Heart Study. Am J Hypertens 8, 657-665.

22. Frontini MG, Srinivasan SR, Xu J, et al. (2008) Usefulness of childhood non-high density lipoprotein cholesterol levels versus other lipoprotein measures in predicting adult subclinical atherosclerosis: the Bogalusa Heart Study. Pediatrics 121, 924-929.

23. Li S, Chen W, Srinivasan SR, et al. (2004) Childhood blood pressure as a predictor of arterial stiffness in young adults: the Bogalusa Heart Study. Hypertension 43, 541-546.

24. Freedman DS, Khan LK, Serdula MK, et al. (2004) Interrelationships among childhood BMI, childhood height, and adult obesity: the Bogalusa Heart Study. Int J Obes 28 , 10-16. 
25. Preis SR, Pencina MJ, Hwang SJ, et al. (2009) Trends in cardiovascular disease risk factors in individuals with and without diabetes mellitus in the Framingham Heart Study. Circulation 120, 212-220.

26. Black RN, Spence M, McMahon RO, et al. (2006) Effect of eucaloric high-and low-sucrose diets with identical macronutrient profile on insulin resistance and vascular risk a randomized controlled trial. Diabetes 55, 3566-3572.

27. Abdulrhman M, El-Hefnawy M, Hussein R, et al. (2011) The glycemic and peak incremental indices of honey, sucrose and glucose in patients with type 1 diabetes mellitus: effects on C-peptide level - a pilot study. Acta Diabetol 48, 89-94.

28. Couch SC, Crandell JL, Shah AS, et al. (2013) Fructose intake and cardiovascular risk factors in youth with type 1 diabetes:
SEARCH for Diabetes in Youth Study. Diabetes Res Clin Pract 100, 265-271.

29. Rovner AJ \& Nansel TR (2009) Are children with type 1 diabetes consuming a healthful diet? A review of the current evidence and strategies for dietary change. Diabetes Educ 35, 97-107.

30. Maahs DM, Dabelea D, D'Agostino RB Jr, et al. (2013) Glucose control predicts 2-year change in lipid profile in youth with type 1 diabetes. I Pediatr $\mathbf{1 6 2}$, 101-107.

31. Ambrosini GL, Oddy WH, Huang RC, et al. (2013) Prospective associations between sugar-sweetened beverage intakes and cardiometabolic risk factors in adolescents. Am J Clin Nutr 98, 327-334. 